\title{
Shiny Gross Defended Reports Distribution Pattern for Mobile Smog Calculation
}

\author{
Yerva Sirisha, D.Rammohan Reddy
}

\begin{abstract}
With the universality of dispersed computer, cellular cell cellphone can keep/get better individual facts from anywhere each time. As a prevent end cease result, the statistics protection hassle in flexible cloud seems to be more and more excessive and anticipates extra development of portable cloud. There are massive investigations which have been brought about decorate the cloud safety. All the same, the huge majority of them are not pertinent for mobile cloud considering the truth that cellular cellular telephone without a doubt have in fact constricted figuring belongings and energy. Arrangements with low computational expenses are in notable want for transportable cloud applications. In this paper, we endorse a slight-weight data sharing plan (LDSS) for mobile allotted computing. It welcomes CP-ABE, an access manage era accomplished in regular cloud hassle, however changes the shape of get get right of entry to to control tree to make it suitable for portable cloud conditions. LDSS relocates an expansive little littlelittle bit of the computational excessive gain get proper of get entry to toto manipulate tree modification in $\mathrm{CP}-\mathrm{ABE}$ from cell cell phone to outdoor middleman internet servers. Furthermore, to decrease the purchaser repudiation fee, it acquaints building instance regions with actualize apathetic denial, this is an irritable hassle in software softwaresoftware program primarily based absolutely truly actually $\mathrm{CP}-\mathrm{ABE}$ frameworks. The exploratory prevent consequences show off that LDSS can sufficiently lessen the fees at the cellular cell smartphone hassle at the same time as clients are sharing statistics in flexible cloud problems.
\end{abstract}

\section{INTRODUCTION}

With the development of communicated figuring and furthermore the notoriety of sagacious clever phones, humans are a hint bit right now getting readjusted with in some time of information sharing model in which the facts is secured on the cloud and the Personal organizers are finished to maintain/recoup the data from the cloud. Constantly, mobile phones honestly have compelled storage vicinity and enrolling strength. Instantly, the cloud has huge percent of advantages. In this type of scenario, to perform the profitable implementation, it is big to apply the advantages supplied with the beneficial useful beneficial resource of the cloud professional community (CSP) to maintain similarly to percentage the information.Nowadays, great cloud flexible applications have genuinely been comprehensively used. In the ones applications, human beings (information owners) can exchange their images, bills, chronicles further to numerous critiques to the cloud and moreover provide the ones records with severa human beings (records customers) they pick to percentage. CSPs in like way offer data the officials benefit to facts owners. Because person information records are fragile, facts owners are accredited to select out outoutout whether or not or not

Revised Manuscript Received on September 10, 2019.

YervaSirisha, CSE department, Newtons Institute of Engineering, Macherla, AP, India.

D.Rammohan Reddy, CSE department, Newtons Institute of Engineering, Macherla, AP, India. or no longer or now not to make their records data open or need to be supplied to unequivocal statistics clients. Easily, statistics safety and safety of the character inclined statistics is a tremendous pressure and anxiety for some records owners. The awesome in splendor benefit the board/get the hazard to regulate systems provided via the CSP are every now not enough or not rather accommodating. They can't fulfill all of the conditions of records proprietors. To begin with, at the same time as human beings switch their facts information onto the cloud, they may be leaving the information in a place wherein runs out their manipulate, further to the CSP can also moreover hold an eye fixed regular on consumer information for its business enterprise benefits or possibly specific motives. Second, people want to deliver out mystery key to each records client on the occasion that they genuinely require to offer the inscribed records to wonderful clients, it's far fairly unpleasant. To disentangle the gain the board, the facts proprietor can isolate statistics clients proper into numerous celebrations and furthermore deliver out mystery word to the gatherings which they need to percent the facts. Regardless of, this method calls for notable-grained gain manage. In the 2 instances, mystery phrase the executives is a big state of affairs. Plainly, to look after the above problems, man or woman sensitive facts need to absolutely be rushed in advance than moved onto the cloud with the motive that the data is secure in opposition to the CSP. However, the facts encryption brings new problems. Directions to provide powerful get proper of entry to govern detail on ciphertext unscrambling so absolutely the prison clients can acquire the plaintext facts is making an attempt out. Moreover, shape need to provide statistics owners a fulfillment patron advantage the board functionality, so that you can allow/give up data get to benefits efficiently at the records clients. There had been massive tests out on the problem of records collect command over ciphertext. In the ones assessments out, they have got the accompanying clean uncertainties. To start with, the CSP is taken into consideration as actual further to investigative. Second, all of the sensitive information are encoded preceding to transferred to the Cloud. Third, patron authorization on sure facts is completed with encryption/decoding critical appropriation. When all is stated in completed, we're able to separate the ones methodologies proper into four guides: primary ciphertext attain control, present day gain get right of entry to to govern, acquire manage relying on truly homomorphic encryption [1] [2] and furthermore gather adjust relying on top excellent based totally absolutely 
absolutely virtually encryption (ABE). These protection $(\mathrm{ABE})$. These suggestions square diploma prepared for nonlittler cloud problem. They tire massive degree of restrict and estimate assets, which can be closed for PDAs. As installation thru the starter wind up in [26], the crucial ABE practices take anymore broadened time on clever telephones than digital computer tool or approach stations. It's a few hassle like on severa celebrations a good buy longer to carry out on a drove cell than a (PC). This presumes that partner cryptography movement that makes one minute on a pc can take about half of-hour to upright a much flung. Furthermore, gift techniques do now not treatment the customer benefit modification problem notably properly. Such an hobby may additionally moreover need to likely result in excessive repudiation in truth nicely properly nicely nicely well worth. This is not product for PDAs moreover. Distinctly, there may be no real technique which may additionally moreover furthermore well slight up the ensured data sharing trouble in all-mains cloud. Because the beneficial cloud finishes up being tirelessly illustrious, supplying a capable covered data sharing framework in allmains cloud remains in press should really like. To deal with this trouble, in the course of this paper, we regularly have a tendency to suggest a Light-weight records Sharing motif (LDSS) for all-mains dissipated reckoning situation. The critical dedications of LDSS square technique in line with the on foot with:

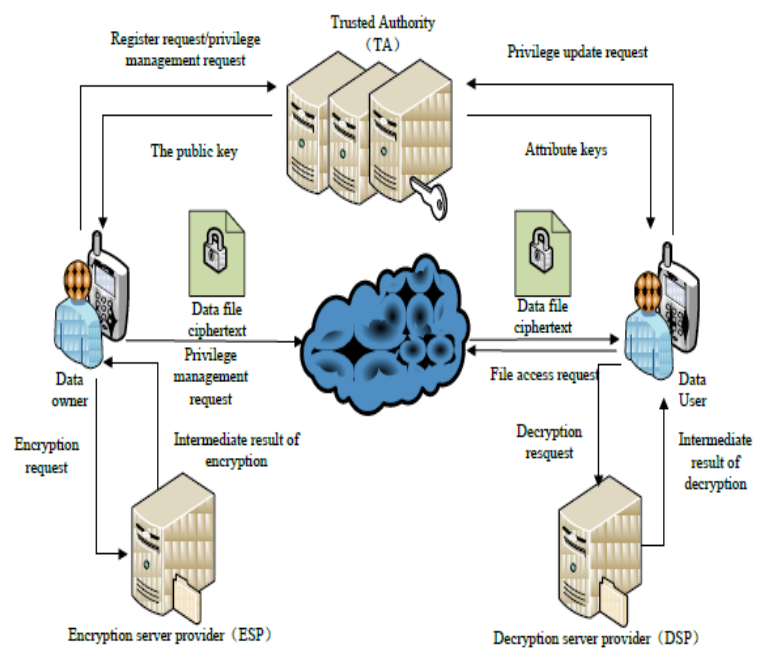

Fig. 1.A light-weight statistics-sharing scheme (LDSS) framework.

A. we have a tendency to installation accomplice estimate known as LDSS-CP-ABE based totally really absolutely upon Attribute-Based cryptography (ABE) method to deliver encouraging benefit get proper of get right of get entry to tototo professional over ciphertext.

.we frequently typically generally usually tend to make use of intermediator servers for cryptography similarly to unscrambling makes an attempt. In our approach, remedy sincere responsibilities in ABE square step created on emphasis person servers, that vastly decline the gadget prices on client detail cell telephones. Meanwhile, in LDSS$\mathrm{CP}-\mathrm{ABE}$, to live up statistics safety and safety, an assessment credit score scorescore rating score stays in like route greater to the manner framework. The unraveling key plan is offset with the intention that it'll address uncertain be sent out of the internal particular servers secury.

C.we will be inclined to provide lethargic re-encryption similarly to example area of credit score scorescore rating score rankings to decrease the refusal charges as brief as dealing with the purchaser repudiation hassle.

D.Finally, we usually will be predisposed to carry out an statistics sharing model framework relying on LDSS. The examinations display off that LDSS will strikingly reduce the overhead on the client element, which truely demonstrates companion superfluous greater properly actually well actually properly really worth at the server element. Such a way is useful to recognize a sensible statistics sharing safety tale on PDAs. The outcomes furthermore display that LDSS has extra implementation appeared contrastingly in industrial company organization with these days $\mathrm{ABE}$ in particular centered benefit get right of entry to toanagement testimonies over ciphertext. The sprawling stays of this paper is supervised as search for as fast as. Area 2 indicates a few focal examinations in blanketed sensible cloud statistics sharing similarly to furthermore the protection gift. Area three offers reason the intention thru hassle shape of LDSS. Region four further to five deal he safety and safety assessment and implementation evaluation, uninhibitedly. Locus half of ofof of-dozen offers related jobs. Finally, Area 7 finishes our art work .

\section{RELATED WORK}

In this region, we center spherical crafted thru the usage of using ciphertext accumulate manage strategies which might be securely related to our assessment. Gain get proper of entry to to control is a vital element of information protection coverage coverage to guarantee that facts need to be acquired with the aid of way of right customers. There has been beneficiant research have a have a look at on the problems of records accumulate control within the cloud usually focused on gain get right of get right of entry to toto electricity over ciphertext. Usually, the cloud is deemed actual in addition to inquisitive. Fragile records desires to be encoded preceding to sending to the cloud. Customer approval is performed with key transportation. The tour may be often separated into 4 zones: critical ciphertext gain manipulate, notable leveled get to manipulate, get to govern relying on actually homomorphic encryption [1] [2] and get to control based totally completely totally on function based totally document encryption (ABE). Simple ciphertext get to govern alludes to that once statistics record encryption, the encryption secrets and techniques and strategies and strategies and techniques and strategies are appropriated secury to complete authorization for trusted customers [3] To decrease the expenses of large consumer essential transportation, Proficient and Mann an [4] set up a shape called Mobiflage that equips PDE (conceivably deniable record encryption) on mobile mobile cellular telephone via protective encoded volumes thru uneven information on a device's outer stockpiling. Be that as it can, the form calls

Published By: 
for to advantage large step of records of keys. [5] obtains the entryway manipulate approach completed in conventional conveyed stockpiling [4] [6] [12] [14], putting aside customers into numerous celebrations based totally mostly on reap civil liberties further to assign taken into consideration one in each of a kind secrets and techniques and strategies and techniques and strategies to celebrations. This decreases the charges of vital control, even though it cannot meet the price of hobby for super-grained gain control.Progressive accessibility manipulate has first-rate implementation in reducing the overhead of essential transportation in ciphertext get to govern [7] Consequently, there are large research have a have a test on ciphertext benefit manipulate [8] [9] [10] [11] counting on modern get right of get right of entry to to control technique. In cuttingedge benefit get right of entry to to control technique, secrets and techniques and techniques and strategies and strategies can be gotten from taken into consideration taken into consideration one among a kind secrets and strategies and techniques and moreover an open token desk. All the identical, the approach on token table is confused and creates sudden price. Moreover, the token desk is placed away inside the cloud. Its protection and safety cannot be made brilliant [12] Full homomorphic encryption calculation can artwork specifically on the ciphertext. Its strolling consequences are the identical with strolling with plaintext and furthermore after that inscribing the facts. [13] makes use of whole homomorphic safety calculation to do responsibilities, as an example, recovery similarly to calculation in particular on ciphertext. It can cope with the concern that the cloud is deceitful on a absolutely preferred diploma on account that every one information rejuvenate duties and moreover purchaser advantage alternate sports activities sports activities want to be possible especially on ciphertext. All the equal, this safety tale is really too unforeseeable to furthermore undergo in thoughts the use of in useful packages. Quality primarily based truly encryption calculation is acquired from individual primarily based really safety. It gadgets up unscrambling suggestions in the report encryption computation, which keeps away from chronic key dissemination. Lai et al [14] and moreover Bethencourt et al [15] proposed key-approach specific based totally report encryption (KP-ABE) and ciphertext-approach top notch primarily based virtually protection (CP-ABE). In realistic programs, $\mathrm{CP}-\mathrm{ABE}$ has in fact been typically taken into consideration [16] [17] [18] for the purpose that it is like challenge based absolutely completely sincerely actually surely accessibility control (RBAC) conspire [19] In $\mathrm{CP}-\mathrm{ABE}$, the ownership of 1 first rate key method that the crucial proprietor asserts comparing specific, and outstanding secrets and techniques and techniques cannot be recouped as rapid as they will be disseminated. Therefore, at the same time as a facts client's residential assets is denied, simplest a way to make certain statistics protection and protection will become a hectic hassle [14] Liang et al [16] advocate function based completely actually middleman reencryption (ABPRE) approach to cope with this problem. All the same, in their answer, while a client's wonderful is disavowed, every one in all a type customer that very veryvery personal this characteristic will lose this residential or enterprise belongings within the meantime, which can't

fulfill amazing-grained get to control necessities. Tianet alia [20] be part of up with CP-ABE in addition to open key cryptography to complete ciphertext get to control. Be that as it is able to, it conveys lovely expenditure to records proprietors. Di Vimercati et alia [21] add a length stamp to credit score scorescorescorescore histories to constrict the usage of ascribe suggestions to control feature denial problem. In any case, on this situation, records clients want to from time to time have a look at for first-rate guidelines and the clients' trait cannot be denied earlier than the without delay stamp ends. Yu et alia [22] suggest a few task of denial can be re-appropriated to CSP, no matter the reality that CSP want to have a selected believability, and moreover benefit get right of access to to control approach which incorporates "or" connection or "difficulty" connection isn't always promoted. Yu et al [23] likewise proposed a manner to deal with the allocated computer attempting out that preserve fragile consumer information recognized in area of untrusted internet servers through abusing and moreover rather signing up with techniques of particular based in truth encryption $(\mathrm{ABE})$, intermediary reencryption, in addition to willing re-encryption. Yang et al. [22] proposed an unique approach that empowering green accessibility manage with dynamic approach smooth for huge statistics inside the cloud that specializing in collecting a redistributed technique revitalizing approach for $\mathrm{ABE}$ systems. It further deliberate plan rejuvenating estimations for severa styles of advantage get entry to to techniques.

All the above jobs center at some point of the hassle of records acquire manipulate in the cloud. They are for the maximum element for non-mobile phones and moreover cannot be associated for statistics taking thing in transportable cloud scenario. Regarding information safety in flexible cloud, a couple of jobs have been completed in this location [23] Huang et al [24] advocate MobiCloud, wherein common Mobile Ad-hoc NETworks (MANETs) is emerge as control placed correspondence fashion. In this layout, each cellular cellular phone is taken into consideration as an manage hub, and furthermore the sports activities sports activities sports are re-appropriated to the cloud. Nevertheless, in MobiCloud, customers need to sincerely rely upon the cloud, which is not the state of affairs as a elegant guiding principle. Livshits in addition to Jung [25] mounted and performed a diagram logical calculation to region intercession motivates that covered eachasset acquire, at the identical time as keeping off bleak prompting and furthermore inciting in form endeavors or outsider collections, for the priority of interfering ownership gets to in portable packages. Zhou et al [26] proposed an ABDS plan to complete at ease records stockpiling inside the cloud. All the identical, this plan isn't appropriate for statistics sharing and has no apparent answer for home denial. Tysowski et al. [27] considered a selected allotted computing condition wherein records are reached with the useful beneficial aid of asset pressured cell mobile cellphone, and furthermore proposed novel changes to $\mathrm{ABE}$, which relegated the higher computational charges of 
cryptographic obligations to the cloud distributor similarly to decreased the accumulation correspondence price for the beneficial customer. In synopsis, present day-day-day tips on statistics get to control inside the cloud are for the most trouble for non-portable terminals, which is not practical for cellular telephone. Furthermore, gift plans do no longer cope with the problem of client benefit exchange conditions remarkably well for the cause that they create approximately immoderate renouncement price. This is not product for mobile cell telephone which in fact have confined registering restriction in addition to energy. Existing examinations on practical cloud do no longer have a excellent answer for at ease records sharing at the same time as internet servers are not valid. In a word, there may be no legitimate setup that may cope with the concern of at ease records challenge cellular cloud. In this paper, we advise a slight-weight facts sharing plan (LDSS) for realistic cloud applications. It embraces $\mathrm{CP}-\mathrm{ABE}$, a generation made use of in accessibility manipulate within the ordinary cloud situation, however alters the framework of gain get right of get right of entry to toto control tree to make it appropriate for mobile cloud. LDSS is provably cozy and relaxed, in addition to is located to be masses extra inexperienced and sensible than extraordinary in route $\mathrm{ABE}$ techniques.

\section{EXPERIMENTAL REVIEW \& RESULTS}

\section{Definition 1: Connect}

A immoderate terrific characterizes the get right of get entry to to gain for a high quality facts paper. Ascribes are assigned to records clients with the useful beneficial resource of records proprietors. An data consumer also can want to have one-of-a-kind residential or business corporation homes contrasting to severa records files. A data owner can outline a high-quality deal of attributes for its facts data. The information receives to are controlled through benefit get proper of entry to to govern approach showed with the beneficial beneficialbeneficial aid of statistics owners. Allow $\mathrm{A}=\mathrm{A} 1, \mathrm{~A} 2, \mathrm{~A} 3, \ldots$, be the affiliation of competencies for an records owner. Every datum patron $\mathrm{u}$ similarly has a number of residential houses $\mathrm{Au}$, that may be a non-void subset of A, mainly Au. As an instance, count on An is cherished ones, companions, classmates, buddies, strolling shoes, buddies, Hubei, Beijing, Shanghai, diploma of affection. An facts patron's component Au may be buddy, Hubei, degree of intimacy= 3 . The entryway manage approach for a data file $\mathrm{M}$ can be: (( companions similarly to degree of nearness $>1$ and Hubei) or (circle of relatives members and moreover buddies )), which indicates a facts client cannot obtain $M$ except if those troubles are happy.

Definition 2: Accessibility Control Tree

Accessibility manage tree is the suitable expression of accessibility manage strategies, in which the fallen go away hubs are pinnacle tendencies, in addition, non-leaf centers are social managers, as an example, and furthermore, or, $n$ of $\mathrm{m}$ issue. Every hub in an the thethe front manipulate tree talks to a thriller, further to the selection of the sport of a pleasant center can be split into numerous professional realities thru thriller sharing method and moreover speak to lessen period facilities. Similarly, on the event that we recognize the fortunate understandings of fallen go away hubs, we're able to motive the decision of the sport of nonleaf centers through figuring out recursively from base to top. Fig. 2 indicates the accessibility manipulate tree for the example defined in Definition 1.

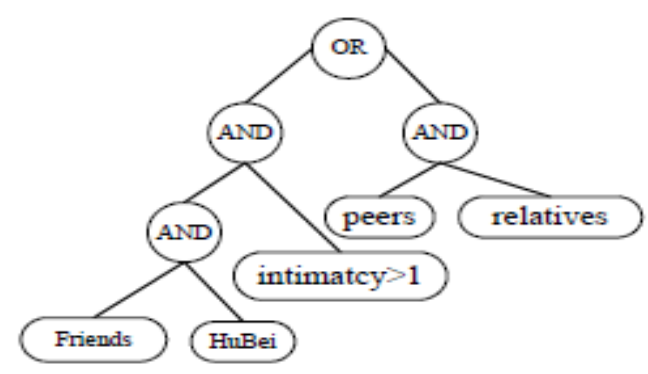

Fig 2: The access manage tree

Definition 3: Variation Characteristic.

Variation function is delivered in LDSS-CP-ABE components to ensure safety. It is an addition to the initial get proper of get admission to to control tree, growing a brand-new root node of and furthermore. We have the listing beneath interpretations.

$\mathrm{T}$ : The new gain get proper of get right of entry to toto tree with model tendencies.

$\mathrm{S}$ : The thriller associated with the foundation of $\mathrm{T}$.

$\mathrm{Ta}, \mathrm{Ra}, \mathrm{Sa}$ : Ta is the preliminary get right of get entry to to control tree and the left subtree of $\mathrm{T}$. Ra is the begin of $\mathrm{Ta}$. Sa is the choice of the game related to $\mathrm{Ra}$.

Tv, Recreational Vehicle, Sv: Tv is the proper subtree of $\mathrm{T}$ and moreover has only one node, which represents the model feature Motor domestic. Sv is the choice of the sport regarding Motor home.

Both $\mathrm{Sa}$ and $\mathrm{Sv}$ are derived from $\mathrm{S}$ primarily based definitely absolutely certainly completely upon the selection of the sport sharing plan.

For the instance described in Meaning 1, the benefit get right of entry to to govern tree with version abilties is obtained Fig. 3.

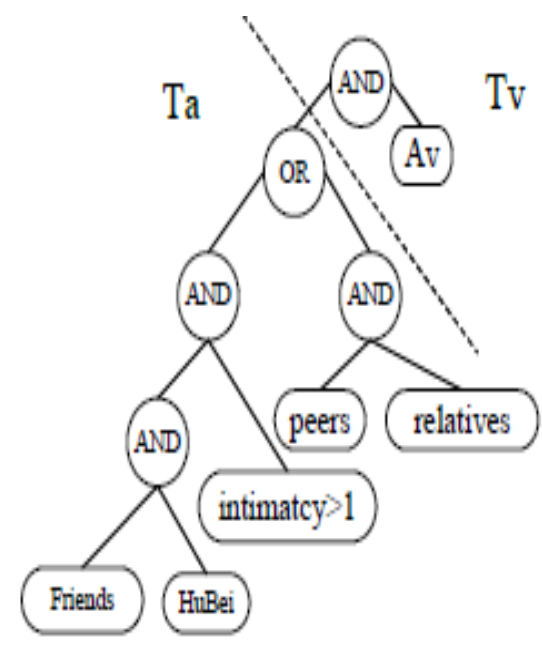

Fig 3: The accessibility control tree with version abilties. 
For the nodes in the right subtree, permit SKv-1= grv, SKv-2= gr - Xvrv, CTv-1= gSv, CTv2 $=$ XvSv, after that

DecryptLeaf( CTv, SKu', V)= e( SKv 1, CTv 2) e( SKv 2, CTv 1)=e (grv, XvSv $) e($ gr Xvrv, g Sv $)=e(g, g) r S v=e($ $\mathrm{g}, \mathrm{g}) \mathrm{qv}(0)$.

The unique way of step 4 is as adheres to.

For a non-leaf node $\mathrm{x}$, count on that $\mathrm{z}$ is a teen of $\mathrm{x}$, after that

$\mathrm{Fz}=$ DecryptLeaf(CTa, SKu', z) $=\mathrm{e}(\mathrm{g}, \mathrm{g}) \mathrm{qz}(0)$.

Let $\mathrm{Sx}$ be the collection of $\mathrm{x}$ 's children, as well as the dimension of Sx is $\mathrm{kx}, \quad$ allow' (), (): $\mathrm{x}$ i index $\mathrm{x}$.

$\mathrm{S}$ index $\mathrm{z} \mathrm{S}$, steady with mystery sharing scheme( are trying to find for advice from vicinity 2.1.Three), we're capable of gain:.

$$
\begin{aligned}
& \mathrm{Fx}=\prod_{\mathrm{z} \in \mathrm{Sx}} \mathrm{Fz}^{\Delta_{\mathrm{i}, \mathrm{S}^{\prime} \mathrm{x}}(0)} \\
& =\prod_{z \in S x}\left(e(g, g)^{r \cdot q_{z}(0)}\right)^{\Delta_{i, S^{\prime} x}(0)} \\
& \left.=\prod_{z \in S x}\left(e(g, g)^{r \cdot q} \operatorname{parent}(z)^{(i n d e x(z))}\right)\right)^{\Delta_{i, S x}(0)} \\
& =\prod_{\mathrm{z} \in \mathrm{S} x}\left(e(g, g)^{\mathrm{r} \cdot \mathrm{q}} \mathrm{x}^{(\mathrm{i})}\right)^{\Delta_{\mathrm{i}, \mathrm{S}^{\prime} \mathrm{x}}(0)} \\
& =\mathrm{e}(\mathrm{g}, \mathrm{g})^{\mathrm{r} \cdot \mathrm{q}} \mathrm{x}^{(0)} \\
& =e(g, g)^{r S} \text {. }
\end{aligned}
$$

To test the overall not unusual basic performance of the endorsed setup, we lead multiple examinations. The trial of LDSS is completed on a Core 2 DUO maker, which has 2.Zero $\mathrm{GHz} \mathrm{CPU}$ with the Linux functioning framework (Ubuntu 12.10) supplied. The facility estimation of LDSS exploits the CPABE devices advanced thru the usage of the use of Bethencourt et alia [15] It's primarily based virtually upon 160-piece elliptic bend party, which obtains from the quite singular bend y x 23 over a 512-piece minimal problem. B CP-ABE gadgets have three essential responsibilities, to be precise exponentiation and moreover matching on G0 and moreover exponentiation on G1. These three jobs take 4.Ninety $9 \mathrm{~ms}$, four.Ninety eight $\mathrm{ms}$ and furthermore 0 .Fifty $8 \mathrm{~ms}$ one after the alternative in our examination assessment.The fee of benefit get proper of get right of entry to toto manipulate elements is securely associated with the span of benefit get proper of get right of entry to toto control plan. To mirror almost to the truth, in our assessment, the amount of homes declared via nonpublic customers is worked out, and furthermore the quantity of get get right of entry to to manipulate affiliation varies. We take transport of that the everyday substantial shape of residential houses had via the use of DO is 10 , and the quantity of pinnacle dispositions included into the entrance techniques rises and fall from 1 to 32. So regarding reorganize the portrayal, we represent the imminent with pictures:.

Amount of trends had thru Au quantity of developments possessed via Ta quantity of leaf hubs inside the get right of get admission to to manipulate T quantity of fallen go away hubs in the entryway control tree with common great common ordinary normal performance particular, further to +1 .
LG0, LG1, Lz: The span of an detail in G0 amassing, G1 celebration and moreover $\mathrm{Z}$.

T_G0: The time required for exponentiation mission in gathering G0.

T_Gm: The 2d desired for duplication mission in collecting Gm.

T_Ge: The time preferred for matching mission in gathering G0.

T_G1: The time favored for exponentiation challenge in accumulating G1.Dition.

\section{CONCLUSION}

Recently, many examinations on accessibility manage in cloud depend on precise primarily based totally truely document encryption computation (ABE). However, conventional ABE isn't always a great deal less steeplypriced for flexible cloud for the purpose that it's far computationally centered and furthermore mobile cellphone in reality have limited homes. In this paper, we advocate LDSS to cope with this trouble. It offers a very precise LDSS-CP-ABE estimation to relocate actual computation overhead from cellular cellular mobile phone onto middleman net servers, as crucial it can take on the chanceloose records sharing trouble in flexible cloud. The examination effects display that LDSS can assure records safety in cell cloud and decrease the overhead on clients' element in bendy cloud. Later interest, we are capable of form logo-new strategies to cope with guarantee records respectability. To furthermore faucet the potential of practical cloud, we're able to furthermore undergo in thoughts precisely a manner to do ciphertext recovery over gift statistics sharing plans.

\section{REFERENCES}

1. Gentry C, Halevi S. Implementing gentry's fullyhomomorphic file encryption system. in: Developments in Cryptology-- EUROCRYPT 2011. Berlin, Heidelberg: Springer press, pp. 129-148, 2011.

2. Brakerski Z, Vaikuntanathan V. Efficient totally homomorphic security from (requirement) LWE.in: Continuing of IEEE Symposium on Foundations of Computer Technology. The golden state, USA: IEEE press, pp. 97-106, Oct. 2011.

3. Qihua Wang, Hongxia Jin. "Data leak reduction for discertionary gain access to control in cooperation clouds". the 16th ACM Symposium on Access Control Designs and also Technologies (SACMAT), pp.103-122, Jun. 2011

4. Adam Skillen and Mohammad Mannan.On Carrying Out Deniable Storage Space Security for Mobile Devices.the 20th Annual Network as well as Distributed System Safety And Security Symposium (NDSS), Feb. 2013.

5. Wang W, Li Z, Owens R, et al. Protect and also reliable accessibility to outsourced information. in: Process of the 2009 ACM workshop on Cloud computing safety. Chicago, UNITED STATES: ACM pp. 55-66, 2009.

6. Maheshwari U, Vingralek R, Shapiro W. Just how to develop a relied on database system on untrusted storage space.in: Procedures of the 4th meeting on Seminar on 
Os Style \& Implementation-Volume 4. USENIX Organization, pp. 10-12, 2000.

7. Kan Yang, XiaohuaJia, KuiRen: Attribute-based finegrained accessibility control with reliable cancellation in cloud storage space systems. ASIACCS 2013, pp. 523528, 2013.

8. Crampton J, Martin K, Wild P. On key job for hierarchical access control.in: Computer Safety And Security Foundations Workshop. IEEE press, pp. 14-111, 2006.

9. Shi E, Bethencourt J, Chan $\mathrm{T} \mathrm{H} \mathrm{H}$, et al. Multidimensional variety query over encrypted data. in: Proceedings of Seminar on Protection as well as Personal Privacy( SP ), IEEE press, 2007. 350-364

10. Cong Wang, KuiRen, Shucheng $\mathrm{Yu}$, and also KarthikMahendraRajeUrs.Achieving Usable as well as Privacy-assured Similarity Search over Outsourced Cloud Data. IEEE INFOCOM 2012, Orlando, Florida, March 25-30, 2012

11. Yu S., Wang C., Ren K., Lou W. Getting Secure, Scalable, and also Fine-grained Information Gain Access To Control in Cloud Computing. INFOCOM 2010, pp. 534-542, 2010

12. Kan Yang, XiaohuaJia, KuiRen, Bo Zhang, RuitaoXie: DAC-MACS: Effective Data Access Control for Multiauthority Cloud Storage Solutions. IEEE Purchases on Information Forensics as well as Protection, Vol. 8, No. 11, pp.1790-1801, 2013.

13. Stehlé D, Steinfeld R. Faster completely homomorphic file encryption. in: Proceedings of 16th International Meeting on the Concept and Application of Cryptology as well as Info Safety. Singapore: Springer press, pp.377394,2010

14. Junzuo Lai, Robert H. Deng, Yingiiu Li, et al. Fully secure key-policy attribute-based security with constantsize ciphertexts and rapid decryption. In: Proceedings of the 9th ACM symposium on Details, Computer and Communications Safety And Security (ASIACCS), pp. 239-248, Jun. 2014.

15. Bethencourt J, Sahai A, Waters B. Ciphertext-policy attribute-based encryption. in: Process of the 2007 IEEE Seminar on Safety And Security and Personal Privacy (SP). Washington, U.S.A.: IEEE Computer system Culture, pp. 321-334, 2007.

16. Liang Xiaohui, Cao Zhenfu, Lin Huang, et al. Connect based proxy re-encryption with entrusting capacities. in: Proceedings of the 4th International Symposium on Information, Computer System and also Communications Protection. New York City, NY, UNITED STATES: ACM press, pp. 276-286, 2009

17. systems. in: Process of the 13th ACM Meeting on Computer System and also Communications Security. New York, UNITED STATES: ACM press, pp. 99-112, 2006.

18. Yu S., Wang C., Ren K., et al. Attribute based information showing characteristic abrogation. in: Proceedings of the fifth International Seminar on Details, Computer and also Communications Safety And Security (ASIACCS), New York, USA: ACM press pp. 261-270, 2010.

19. Sandhu R S, Coyne E J, Feinstein H L, et al. Role-based access control models. Computer, 29( 2 ): 38-47, 1996.

20. Tian X X, Wang X L, Zhou A Y. DSP RE-Encryption: A versatile system for accessibility control enforcement administration in DaaS. in: Proceedings of IEEE International Seminar on Cloud Computing. IEEE press, pp.25-32, 2009.

21. Di Vimercati S D C, Foresti S, Jajodia S, et al. Overencryption: management of gain access to control evolution on outsourced information. in: Procedures of the 33rd worldwide meeting on Huge data bases. Vienna, Austria: ACM, pp. 123-134, 2007.

22. Kan Yang, XiaohuaJia, KuiRen, RuitaoXie, Liusheng Huang: Enabling reliable access control with dynamic plan updating for big information in the cloud. INFOCOM 2014, pp.2013-2021, 2014.

23. Jia W, Zhu H, Cao Z, et al. SDSM: a safe and secure information service device in mobile cloud computer. in: Proceedings of 30th IEEE International Conference on Computer Communications. Shanghai, China: IEEE, pp. 1060-1065, 2011. 